1 Title:

2

3

4

5

6

7

8

9

10

11

12

13

14

15

16

17

18

19

20

21

22

\title{
Weighted trait-abundance early warning signals better predict population collapse
}

4

Authors: Christopher F. Clements ${ }^{1,2} *$, Martijn van de $\mathrm{Pol}^{3,4}$, Arpat Ozgul $^{1}$

\section{Affiliations:}

${ }^{1}$ Department of Evolutionary Biology and Environmental Studies, University of Zurich, Zurich 8057, Switzerland.

${ }^{2}$ School of BioSciences, University of Melbourne, Melbourne 3010, Australia.

3 Department of Ecology \& Evolution, Research School of Biology, Australian National University, Canberra 2601, Australia.

${ }^{4}$ Department of Animal Ecology, Netherlands Institute of Ecology (NIOOKNAW), Wageningen 6708PB, the Netherlands.

Keywords: Body size, extinction, population dynamics, tipping points, trait dynamics

Word count: 2489

\section{Acknowledgements:}

This work was made possible by an SNSF Post-doctoral fellowship (P300PA_174359/1) awarded to C.C. and an ERC Starting grant (\#337785) to A.O. M.vd.P. was supported by a Future fellowship from the Australian Research Council (FT12010020). 


\section{Abstract:}

24 Predicting population collapse in the face of unprecedented anthropogenic 25 pressures is a key challenge in conservation. Abundance-based early warning signals 26 have been suggested as a possible solution to this problem; however, they are known 27 to be susceptible to the spatial and temporal subsampling ubiquitous to abundance 28 estimates of wild population. Recent work has shown that composite early warning 29 methods that take into account changes in fitness-related phenotypic traits - such as 30 body size - alongside traditional abundance-based signals are better able to predict 31 collapse, as trait dynamic estimates are less susceptible to sampling protocols. 32 However, these previously developed composite early warning methods weighted the 33 relative contribution of abundance and trait dynamics evenly. Here we present an 34 extension to this work where the relative importance of different data types can be 35 weighted in line with the quality of available data. Using data from a small-scale 36 experimental system we demonstrate that weighted indicators can improve the 37 accuracy of composite early warning signals by $>60 \%$. Our work shows that non38 uniform weighting can increase the likelihood of correctly detecting a true positive 39 early warning signal in wild populations, with direct relevance for conservation management.

Keywords: 
46

47

48

49

\section{Introduction}

Statistical early warning signals (EWSs) calculated from abundance time series data have been suggested as a possible method for predicting approaching population collapses and regime shifts (Drake \& Griffen, 2010; Carpenter et al., 2011; Dakos et al., 2012; Kéfi et al., 2013). However, abundance-based early warning signals are known to be susceptible to the spatial and temporal subsampling ubiquitous to wild population abundance estimates (Clements et al., 2015), and have been criticized for not reliably predicting significant declines in natural populations (Burthe et al., 2016). Recent work has sought to resolve these issues by incorporating data on the dynamics of fitness-related phenotypic traits alongside abundance data (Clements \& Ozgul, 2018). Traits such as body size are highly responsive to environmental perturbations and changes in the dynamics of these traits often precede demographic responses to deteriorating environments (Anderson et al., 2008; Ozgul et al., 2014; Clements \& Ozgul, 2016a). Incorporating information on the shift in the body-size distribution of a population can not only provide an additional measure of stability (Anderson et al., 2008), but has the potential to improve the predictive accuracy of EWS as trait dynamic estimates may be less susceptible to sampling protocols than population abundance estimates are when the distribution of ages and sexes is assumed to be random (spatial partitioning between ages or sexes may affect this) (Clements et al., 2015, 2017; Clements \& Ozgul, 2016a). Previous work has shown composite early warning metrics that include data on both abundance and trait dynamics better predict population collapse than those that incorporate abundanceonly or trait-only data (Clements \& Ozgul, 2016a). 
71 statistical signals are normalized and then summed to create a single composite signal.

72 Clements \& Ozgul (2016a) used this approach to incorporate shifts in mean body size

73 and variance in body size along with concurrent changes in the statistical properties of

74 an abundance time series, and demonstrated that such an approach can significantly

75 improve the reliability of early warning signals in both experimental (Clements \&

76 Ozgul, 2016a) and natural (Clements et al., 2017) populations. However, in this

77 method the relative importance of abundance versus trait data in the composite

78 indicators was weighted evenly. Given the known issues with abundance data, a

79 logical extension to this method is to non-evenly weight the relatively importance of

80 abundance and trait data in the composite indicators.

81 Non-uniform weighting of model parameters has a history of use in

82 conservation biology, particularly in determining optimal management strategies to

83 maximize outputs from limited resources (Joseph, Maloney \& Possingham, 2009).

84 For example habitat conservation may be prioritized based on the suitability of the

85 habitat for certain species, with such weightings often being determined by expert

86 opinion (Lehtomäki et al., 2009). Such approaches have also been used to assess

87 trade-offs, for example between conservation and carbon sequestration (Thomas et al.,

88 2013). As well as expert opinion, weighting may be based on more quantitative

89 measures of data quality; for example by the frequency of sampling of a population to

90 estimate abundances, or the percentage of a habitat sampled when counting

91 individuals, both of which have been shown to affect the reliability of early warning

92 signals (Clements et al., 2015). However, practitioners must first discern if non-

93 uniform weightings convey an advantage before implementing such an approach for

94 wild populations. 

reanalyze the data from an experimental protist microcosm system, presented in

101 Clements \& Ozgul (2016a), and show that alternate weightings can improve the predictive ability of composite EWS by decreasing the frequency of false positive signals, and increasing the frequency of true positive signals.

104

\section{Methods}

Data on the population dynamics and body-size (width, $\mu \mathrm{m}-\mathrm{a}$ proxy for mass) of individuals of a predatory ciliate protozoa (Didinium nasutum) feeding on a

109 bactiverous ciliate protozoa (Paramecium caudatum) were collected over a 47-day

110 period (Fig. 1). Populations of D. nasutum were subjected to four different treatments

111 (15 replicates per treatment), where the number of $P$. caudatum fed to each population

112 per day was manipulated. In one treatment ("Constant") populations of D. nasutum

113 were fed 300 P. caudatum per day for the 47 days of the experiment, whilst in the

114 other three treatments the number of $P$. caudatum declined through time at three

115 different rates ("Slow”, "Medium", "Fast") driving the populations of D. nasutum to

116 extinction at varying points in time, and with varying population dynamics prior to

117 extinction (Fig. 1). None of the populations in the Constant treatment went extinct.

118 For each population the time at which it passed through a tipping point, if at all, was 119 calculated (as in Drake \& Griffen, 2010), and early warning signals were then 
120 calculated prior to the occurrence of each of these tipping points. Because of the size

121 of the microcosms it was impractical to count every individual of a population, hence

122 a subsample was taken (10\% of the habitat, a volume that allowed all individuals to

123 be easily counted with close to zero error) and we assumed that the total number of

124 individuals in each microcosm was reflected by the abundance in the subsample.

125 Whilst this undoubtedly introduced some minor error into the abundance estimates,

126 EWSs were still detectable using this uncorrected subsample data (Clements \& Ozgul,

127 2016a). We believe that this uncertainty in abundances is very representative of the

128 ubiquitous spatial subsampling associated with the monitoring of all wild populations,

129 and hence applying such methods to this data is a reliable reflection of the challenges

130 of applying them to real world population dynamics. For full details of the

131 experimental design and protocols see Clements \& Ozgul (2016a).

132

133 Early warning signals

134 Previous work has identified a composite index comprised of the coefficient of

135 variation of the abundance time series $(c v)$, shifts in mean body size of the individuals

136 in the population (size), and shifts in the standard deviation of mean body size

137 (sd.size) as producing the most reliable estimates of whether a population was at risk

138 of collapse in these experimental data (Clements \& Ozgul, 2016a). Here we test this

139 composite index by systematically altering the weighting of these three competent

140 parts as a proof of concept of non-uniform weighting increasing the predictive

141 accuracy of the composite metric.

142 Here we implement the approach developed by Clements \& Ozgul (2016a).

143 Each of the three leading indicators ( $c v$, size, sd.size) was calculated at each day

144 observations were made, and for each experimental population independently. Each 
145 leading indicator was then normalized by subtracting the long-run mean of that

146 indicator from the value of that indicator at each time point, and dividing it by the

147 long run standard deviation (Drake \& Griffen, 2010; Clements \& Ozgul, 2016a)

148 (Supplementary Information). The value of the composite early warning signal was

149 then calculated by summing the value of each leading indicator ( $c v$, size, sd.size $)$ at

150 each time point. Previous work has suggested an EWS could be considered present

151 when the value of this composite EWS exceeds its running mean by either 1 or $2 \sigma$

152 (Drake \& Griffen, 2010). Recent evidence suggesting a $2 \sigma$ threshold provides more

153 reliable results (Clements \& Ozgul, 2016a) and consequently here we consider a

154 signal present at a $2 \sigma$ threshold.

155 The weighting of each of the three leading indicators was altered by

156 multiplying the normalized value of each metric prior to summing them together to

157 calculate the composite EWS. Each leading indicator was weighted from 1 to 10, with

158 every combination of weightings tested (e.g. $c v_{w}=1$ : size $_{w}=2$ : $s i z e . s d_{w}=5$,

$159 c v_{w}=8:$ size $_{w}=4:$ size.s $\left.d_{w}=1\right)$. The performance of each weighting was assessed by using

160 a "normalized metric score" (Clements \& Ozgul, 2016a), calculated by subtracting the

161 proportion of false positives (EWS present in data from the constant treatment) from

162 the proportion of true positives (EWS present in data from the slow, medium, and fast

163 treatments). The highest scoring weighting for each of the slow, medium, and fast

164 treatments was compared to uniform weighting in each of these treatments (Fig. 2a).

165 The best metric when data from all three treatments were grouped together was

166 calculated as the weighting with the highest normalized metric score, and the

167 minimum difference in normalized metric scores between treatments (Fig. 2b). This

168 gave an indication as to the weighting that was most robust to different rates of 
169 environmental change, and thus potentially most widely applicable to different 170 scenarios.

171 All analyses were carried out using the statistical software R (R Development

172 Core Team, 2016), and the code to implement weighted trait-abundance early warning

173 signals is available as supplementary information.

174

175 Results

176 Experimental data

177 Non-uniform weighting of the importance of abundance and trait data in early

178 warning indicators can improve the reliability of these methods in predicting

179 population collapses (Fig. 2). The largest improvement (62.5\%) was seen when data

180 from the Medium treatment was analyzed, possibly because uniform weighting

181 performed relatively poorly (Fig. 2a). The highest achieved normalized metric score

182 was 0.8 (in the slow treatment), suggesting very high numbers of true positive EWS,

183 and low numbers of false positive EWS (Fig. 2a).

184 When data from three deteriorating treatments was grouped together the

185 weighting that produced the greatest improvement in predictive accuracy weighted

186 the relative importance of $c v$, size.sd, size as 4:7:4, although the improvement over

187 uniform weighting was not large (Fig. 2b), suggesting that the how fast the pressure

188 on the system changes (known as the rate of forcing (Clements \& Ozgul, 2016b)) may

189 be an important factor in determining not only the correct weighting to apply, but

190 also our ability to reliably predict population declines. To highlight this, the 4:7:4

191 weighting performed as well as the best weighting in the fast treatment, average in the

192 medium treatment, and worse than both the uniform and best weighting in the slow

193 treatment (Fig. 2a). 


\section{Discussion}

197 biology. Because previously developed EWS that take into account both trait and

198 abundance data are non-system specific, and thus widely applicable, they may be of 199 particular interest. Here we analyze data from a small-scale experimental system and 200 show that non-uniform weighting can improve the reliability and strength of trait201 abundance early warning signals, but that the strength of this improvement is not uniform across different rates of environmental change.

203 Previous work in small-scale experimental systems has identified a composite 204 metric of $c v$, size, and size.sd as the most reliable predictor of population collapse in 205 experimental microcosm populations. This method provides improved reliability over 206 methods that are based on either abundance-only or size-only data; however, the 207 method still produces false positive and false negative signals in some populations. 208 Because of the known susceptibility of abundance-based early warning signals to poor 209 quality data (Clements et al., 2015), non-uniformly weighting the components of 210 composite metrics provides an obvious extension to this previous work.

211 Here we demonstrate, using the same experimental data with which the 212 original trait-abundance method was developed, that a weighting of 4:7:4

213 (cv:size.sd:size) provides the greatest overall improvement across all three treatments, 214 with the minimum between-treatment variation in this result (Fig. 2b). Resilience to 215 treatment variation in performance in the experiment is important, as it maximizes the 216 reliability of applying such methods to systems where the rate of change remains 217 unknown. However, the among-treatment variation in the potential advantages of 218 non-uniform weighting should not be ignored, as with weightings other than 4:7:4 
219 there were significantly higher normalized metric scores in the medium and slow

220 treatments (Fig 3a). Such a result is likely to be driven by the rate of forcing, known

221 to potentially alter the detectability of EWSs (Clements \& Ozgul, 2016b), of the

222 system altering the rates of change of the mean and $\sigma$ body size of individuals. For

223 example, mean body size rapidly declines in the fast treatment (Fig. 1), whilst in the

224 medium treatment body size decline is more gradual and a weighting towards the

225 coefficient of variation of abundance, rather than towards body size, improves

226 predictive accuracy (Fig. 2). These results suggest that the rate of forcing a system

227 can alter the weighting that produces the most reliable predictions of an approaching

228 population collapse.

229 Generalizing such a result to real-world systems may be problematic, as we

230 cannot assume that the population and trait dynamics of the microcosm system

231 analyzed here are truly representative of all real-world population collapses. Ideally

232 one would select the weighting based on an estimate of the reliability of the available

233 abundance or trait data, and possibly based on the rate of forcing of the system,

234 although doing so is likely to be non-trivial. If, for example, available abundance data

235 are known to be estimated from a survey conducted on a small proportion of the

236 known range of a species, or are temporally limited, it may be prudent to calculate the

237 presence of early warning signals with a bias in favor of trait-based data. A less

238 quantitative option would be to weight metrics based on expert opinion of the

239 reliability of the available data. However, whilst criticism has been leveled at the use

240 of expert opinion in conservation management, it has been shown to be useful if

241 approached with caution (Johnson \& Gillingham, 2004; Martin et al., 2005, 2012).

242 Similar caution must be applied to the non-uniform weighting of trait and abundance

243 data in the models presented here, however the significant improvements in the 
244 predictive accuracy of these approaches when weighting is non-uniform mean that

245 canvasing expert opinion may be a relatively simple and cost-effective solution to

246 improve predictive accuracy.

247 In conclusion, we demonstrate the possible advantages of non-uniform

248 weighting in an early warning signals framework. This work provides a first step to

249 improving the reliability of recently proposed abundance-trait methods (Clements \&

250 Ozgul, 2016a), and may be used to negate some of the known issues that affect

251 abundance-based EWSs (Clements et al., 2015). Future work may seek to make more

252 concrete recommendations on weightings based on qualitative measures such as

253 expert opinion, or more quantitative measures such as measures of data quality, the

254 known level of threat to a species or a population, the trophic level of the species, or

255 its connectedness in a network. One option to tackle this is to use complex size-

256 structured community models, such as those commonly used in fisheries (Blanchard

257 et al., 2012; Scott, Blanchard \& Andersen, 2014), to simulate shifts in the trait

258 dynamics and abundances of multiple interacting species, allowing alternative

259 weightings of data from various trophic levels to be tested on communities where

260 collapse can be invoked by, for example, overfishing or changing climatic variables.

\section{References}

263 Anderson CNK., Hsieh C., Sandin S a., Hewitt R., Hollowed A., Beddington J., May

264 RM., Sugihara G. 2008. Why fishing magnifies fluctuations in fish abundance. $265 \quad$ Nature 452:835-9.

266 Blanchard JL., Jennings S., Holmes R., Harle J., Merino G., Allen JI., Holt J., Dulvy 267 NK., Barange M. 2012. Potential consequences of climate change for primary 268 production and fish production in large marine ecosystems. Philosophical 

367:2979-89. DOI: 10.1098/rstb.2012.0231.

271 Burthe SJ., Henrys PA., Mackay EB., Spears BM., Campbell R., Carvalho L., Dudley B., Gunn IDM., Johns DG., Maberly SC., May L., Newell MA., Wanless S., Winfield IJ., Thackeray SJ., Daunt F. 2016. Do early warning indicators consistently predict nonlinear change in long-term ecological data? Journal of Applied Ecology 53:666-676. DOI: 10.1111/1365-2664.12519.

Carpenter SR., Cole JJ., Pace ML., Batt R., Brock W a., Cline T., Coloso J., Hodgson JR., Kitchell JF., Seekell D a., Smith L., Weidel B. 2011. Early Warnings of Regime Shifts: A Whole-Ecosystem Experiment. Science (New York, N.Y.) 332:1079-1082.

283 Clements CF., Drake JM., Griffiths JI., Ozgul A. 2015. Factors influencing the detectability of early warning signals of population collapse. Am Nat 186:50-58. DOI: in press.

Clements CF., Ozgul A. 2016a. Including trait-based early warning signals helps

Clements CF., Ozgul A. 2016b. Rate of forcing and the forecastability of critical transitions. Ecology and Evolution 6:7787-7793.

291 Clements CF., Ozgul A. 2018. Indicators of transitions in biological systems. Ecology $292 \quad$ Letters In press.

293 Dakos V., Carpenter SR., Brock WA., Ellison AM., Guttal V., Ives AR., Kéfi S., 
294 Livina V., Seekell DA., van Nes EH., Scheffer M. 2012. Methods for detecting

295 early warnings of critical transitions in time series illustrated using simulated

296 ecological data. PloS one 7:e41010. DOI: 10.1371/journal.pone.0041010.

297 Drake J., Griffen B. 2010. Early warning signals of extinction in deteriorating 298 environments. Nature 467:456-459.

299 Johnson CJ., Gillingham MP. 2004. Mapping uncertainty: Sensitivity of wildlife 300 habitat ratings to expert opinion. Journal of Applied Ecology 41:1032-1041.

301 Joseph LN., Maloney RF., Possingham HP. 2009. Optimal allocation of resources 302 among threatened species: a project prioritization protocol. Conservation Biology 303 23:328-338. DOI: 10.1111/j.1523-1739.2008.01124.x.

304 Kéfi S., Dakos V., Scheffer M., Van Nes EH., Rietkerk M. 2013. Early warning 305 signals also precede non-catastrophic transitions. Oikos 122:641-648. DOI: $306 \quad$ 10.1111/j.1600-0706.2012.20838.x.

307 Lehtomäki J., Tomppo E., Kuokkanen P., Hanski I., Moilanen A. 2009. Applying 308 spatial conservation prioritization software and high-resolution GIS data to a 309 national-scale study in forest conservation. Forest Ecology and Management 310 258:2439-2449. DOI: 10.1016/j.foreco.2009.08.026.

311 Martin TG., Burgman MA., Fidler F., Kuhnert PM., Low-Choy S., Mcbride M., 312 Mengersen K. 2012. Eliciting Expert Knowledge in Conservation Science. 313 Conservation Biology 26:29-38.

314 Martin TG., Kuhnert PM., Mengersen K., Possingham HP. 2005. The power of expert 315 opinion in ecological models using Bayesian methods: Impact of grazing on 316 birds. Ecological Applications 15:266-280. DOI: 10.1890/03-5400.

317 Ozgul A., Bateman AW., English S., Coulson T., Clutton-Brock TH. 2014. Linking 318 body mass and group dynamics in an obligate cooperative breeder. Journal of 
Animal Ecology.

320 R Development Core Team. 2016. R: A language and environment for statistical 321 computing.

322 Scott F., Blanchard JL., Andersen KH. 2014. mizer $\square$ : an R package for multispecies, 323 trait-based and community size spectrum ecological modelling. Methods in 324 Ecology and Evolution 5:1121-1125. DOI: 10.1111/2041-210X.12256.

325 Thomas CD., Anderson BJ., Moilanen A., Eigenbrod F., Heinemeyer A., Quaife T., 326 Roy DB., Gillings S., Armsworth PR., Gaston KJ. 2013. Reconciling biodiversity and carbon conservation. Ecology Letters 16:39-47.

328 

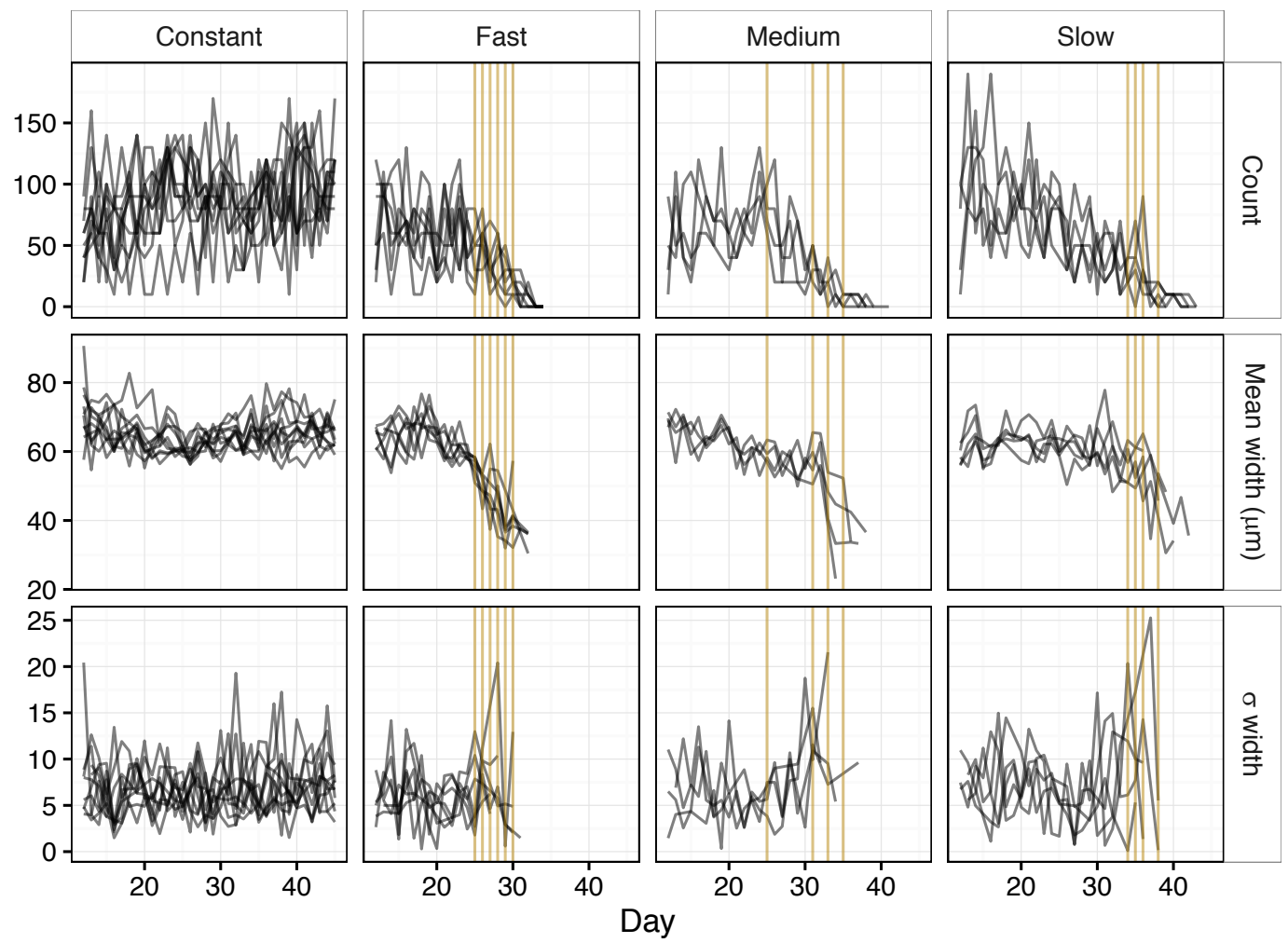

Figure 1. Black lines describe the population and body size dynamics of individual populations of Didinium nasutum subjected to four different experimental treatments (constant, fast, medium, and slow rates of decline in prey availability). Data from day 0 to 12 were removed to minimize the effects of transitory dynamics. Each vertical gold line indicates an inferred tipping points for a collapsing population. 

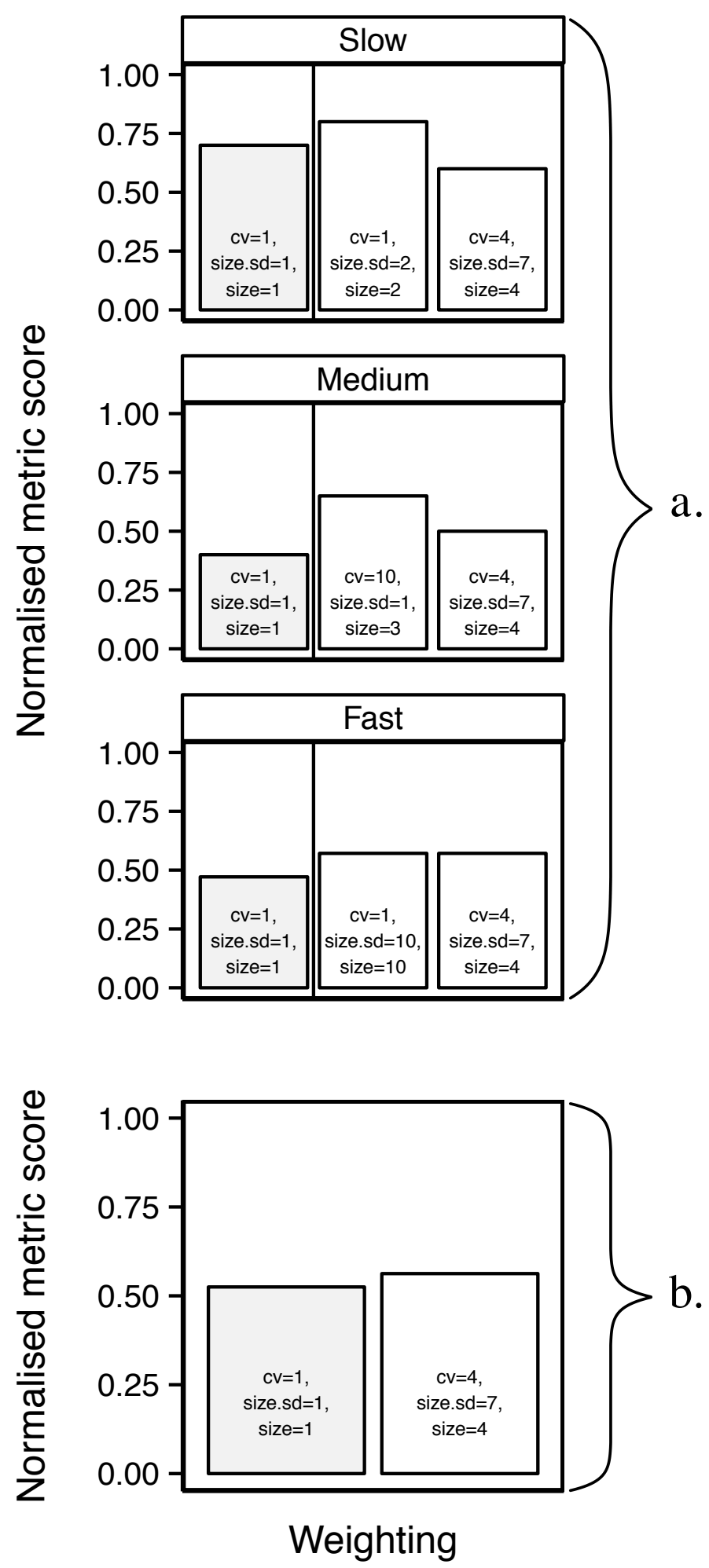

Figure 2. (a) The highest scoring weighting across each of the experimental treatments compared to even weighting and the best weighting when data from all treatments were combined, and (b) the weighting with the highest normalized metric score across all three treatments, and the lowest difference in normalized metric score amongst treatments. 